\title{
TESTS OF EVOLUTION MODELS USING STAR CLUSTERS
}

\author{
J.-C. MERMILLIOD \\ Institut d'Astronomie, Université de Lausanne \\ CH-1290 Chavannes-des-Bois
}

\section{Introduction}

The fitting of isochrones over star-cluster colour-magnitude diagrams is a favourite test of stellar evolution models because it seems to be the easiest and most evident one: the star distribution in the colour-magnitude diagram represents a constant age locus, provided the clusters are old enough and the internal age dispersion is negligible. The ratios of evolved to mainsequence stars in open clusters, or of population along the red giant branch in globular clusters, could provide valuable tests, but it is difficult to estimate these ratios with small enough uncertainties. Furthermore the detailed surface chemical composition (element abundances and isotope ratios) is not known for many cluster stars and model predictions cannot be tested in various environments.

Tests have been mostly devoted to the main sequence and the long debate between canonical precepts and core overshooting is still active. Stothers (1991) has argued that the use of the new opacities (Rogers \& Iglesias 1992) reduces the necessity of adding overshooting to the models. Castellani et al (1992) computed extensive grids of models with canonical precepts. However, recent papers (Carraro et al. 1993; Meynet et al. 1993; Demarque et al. 1994) show that a moderate core overshooting (about 0.2 $\mathrm{Hp}$ ) is still necessary. Basically, cluster observations call for more massive cores, whatever process is at work. An excellent review of the various ingredients included in models (equation of state, nuclear rates, opacities, convection) and the effects of their changes has been presented by Vandenberg (1991).

The fit quality depends on the somewhat subjective interpretation of the cluster upper main sequence in presence of binaries and non-members. However, few clusters have enough data to really describe the turnoff mor- 
phology: accurate photometry, membership probabilities from proper motion studies and radial velocities to identify the binaries. Extensive radial velocity studies are in progress for four clusters: M67 (Mathieu et al. 1990), NGC 752 (Daniel et al. 1994), and NGC 3680 and IC 4651 (Nordström \& Andersen 1994). Many data have however not been published yet.

\section{Problems}

\subsection{DATA QUALITY AND RELIABILITY}

The existence of high-quality photometric data is the first condition required to perform reliable tests. Although modern CCD observations may appear as free of errors, a few examples show that CCD data should be used with much care because severe systematic errors may happen. An external quality check is not feasible for many clusters: only 11 among 72 open clusters in my database (Mermilliod 1992) have presently data from more than one source.

\subsection{THE SAMPLE COMPLETENESS}

Due to the often small size of CCD fields, it is tempting to observe only the central part of unstudied clusters to define their main characteristics at low cost. This policy results in a dramatic loss of information, because, for example, stars of different masses show different degrees of concentration towards the cluster centers. To test models of stellar evolution, samples of stars as complete as possible are needed.

\subsection{ZAMS DEFINITION}

The ZAMS defined by Schmidt-Kaler (1982) is well adapted to describe the lower main sequence of the Hyades and Praesepe, but not that of the Pleiades. It is useful for stars more metal-rich than the Sun and closer to the Hyades' metallicity. One would ideally need several ZAMS, accounting for the differences in chemical composition. However, most tabulations or formulas to adapt the ZAMS are based on models rather than on observations.

\subsection{RELATION $\mathrm{T}_{\mathrm{EFF}}$ - COLOUR INDICES}

At the moment the worst problem I see is the uncertainty in the relations between $T_{\text {eff }}$ and colour indices, both for solar and non-solar chemical compositions. Available tables have separate entries for the different luminosity classes, but do not provide information for different chemical compositions. Transformations based on empirical data or on Kurucz models are not ex- 
actly equivalent. Because the transformations for non solar composition are mostly based on Kurucz models, can we trust the results? We are caught in a loop and there is a dramatic need for fundamental data to fix the temperature scale.

As a result, the transformations from theoretical temperature to observed parameters (colour indices) used by several groups are different, with the following consequence: although models are quite similar in the

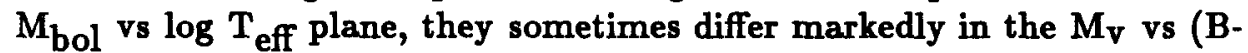
V)o plane. The ages or star parameters deduced from the various models may therefore differ significantly as a direct effect of the calibration used.

\section{Conclusions}

As long as we combine (1) uncertainties on temperature transformation, (2) systematic photometric errors, (3) uncertainties on cluster reddening and distances, good agreement will most often be found between models and observations because there are too many free parameters. To make meaningful comparisons, the values of the reddening and distance should be used without further adaption. This is possible if, and only if, we have correct tables of transformation, used by most groups computing models.

We need more complete, high-precision studies of those star clusters that are really suitable for such comparisons, as well as a full coverage of the cluster area (not only the center) to test the population density predictions and surface abundances and more information on cluster-star properties (rotation, binarity, variability).

Beyond the general agreement, there are reasons not to be satisfied with the present state of the art, because we attribute too much confidence in processes that test models with information produced by or extracted from other models. Direct observations are difficult to obtain and it is easier to rely on models that inspire confidence.

\section{References}

Carraro G., Bertelli G., Bressan A., Chiosi C. 1993, A\&AS 101, 381

Castellani V., Chieffi A., Straniero O. 1992, ApJS 78, 517

Daniel S.A., Latham D.W., Mathieu R.D., Twarog B.A. 1994, PASP 106, 281

Demarque P., Sarajedini A., Guo X.-J. 1994, ApJ 426, 165

Mathieu R.D., Latham D.W., Griffin R.F. 1990, AJ 100, 1859

Mermilliod J.-C. 1992, Bull. Inform. CDS 40, 115

Meynet G., Mermilliod J.-C., Maeder A. A\&AS 98, 477

Nordström B., Andersen J. 1994, (in preparation)

Schmidt-Kaler Th. 1982, in Landolt-Börnstein, New Series, group VI, vol. 2b, eds K. Schaifers \& H.H. Voigt (Berlin, Springer Verlag)

Stothers R.B. 1991, ApJ 383, 820

Vandenberg D. 1991, ASP Conf. Ser. 13, 185 
R. GRAY to D. DRAVINS Q. Although you concentrated on the effects of rotation which can be observed in high resolution spectra, I would like to point out that quite significant effects of rotation due to the variation of $\mathrm{T}_{\text {eff }}$ and $\log \mathrm{g}$ across the surface of the rotating star can be observed in MK dispersion spectra, and even in photometry such as Stromgren and Geneva photometry.

A. Stellar rotation can indeed be detected and studied through spectroscopic, photometric or polarimetric monitoring, identifying the rotational period from the modulation of e.g. photometric indices (reflecting poleequator differences) or in e.g. chromospheric emission lines (reflecting the rotation of active region). Also splitting patterns in the temporal spectra of stellar oscillations may provide a seismologic measure of stellar rotation. Following the invitation by the organizers however, my review was limited to the determination of $v$ sini from single-epoch spectroscopic observations.

A.T. YOUNG to D. DRAVINS Q.Grating spectrometer have an inherently asymetric profile when one is concerned with effects below $1 \%$ in relative intensity, as for many of the effects you have shown. For these weak effects, one should probably use a Fourier - transform spectrometer instead of a grating instrument.

A. It is perfectly possible to detect and also rather accurately measure e.g. weak subrotational features with ordinary grating spectrometers. Possible improvements when using Fourier-transform spectrometers are more related to systematic effects on e.g. spectrum continum levels, wavelength scales or achievable resolutions. For a recent review of such problems, see e.g. my paper "Instrumental Effects in Stellar Spectroscopy", in C. Sterken and M. de Groot, eds: "The impact of long term monitoring in variable stars research", Kluwer, p. 269 (1994).

B. SCHLESINGER to ANDERSEN/MERMILLIOD Q. In the first item that you have shown on the list of the things to be done, I would expand the color-temperature conversion term to include colors other than B and V, toward the ultraviolet and the red and infrared, for use in studing young clusters and evolved stars. I would also add bolometric corrections as a function of temperature to the color-temperature conversions as a needed term.

A. Yes I would agree (and add intermediate-band colors).

A.T. YOUNG to ANDERSEN Q. I'am glad you called attention to systematic errors in photometry. Unless the instrumental passband are very 
close the standard ones, the conventional transformation will leave substantial systematic errors for reddened stars and other that differ from the standard stars in metallicity.

A. Yes, I believe my diagrams spoke by themselves. After spending six years on radial velocities it was not a welcome surprise to find that the photometry had to be done all over again!

R. KRAFT to J. ANDERSON Q. The Mazzei and Pigatto introduction of rotation and overshooting resolved the discrepancy between contraction and nuclear clusters time scale.

What is the present status of this result at the present time especially in the light of your studies?

A. I'm not aware of any new work on this subject. However, the dynamics of the cluster itself will, of course, have to be restudied with the improved membership information, and the earlier factor- 2 discrepancy between ages from standard and overshooting models has now narrowed down to some $20 \%$.

P. MAXTED to ANDERSEN/MERMILLIOD Q. Is there is a natural place for fiber feed instruments in this field and are instrument builders being made aware of this?

A. Yes, and this was part of the motivation for building a multislit spectrograph on the NTT.

I. PLATAIS to ANDERSEN/MERMILLIOD Q. I don't think the saga on the open cluster NGC 3680 is over. Very recently we completed a proper motion study of NGC 3680, results of which are submitted for publication in A.J.. In some cases we noticed a discrepancy between the proper motion membership v.s. radial velocity membership. Before drawing the final conclusions we must understand the cause of that discrepancy. In addition to the uncertainties in observationnal data I would like to add natural limitations set by applying the proper motion criterion in membership analysis. One must be critical analysing proper-motion data in both respects the possible systematic errors and the interpretation of membership probability numerical values. 